\title{
Evaluation of trends of drug-prescribing patterns based on WHO prescribing indicators at outpatient departments of four hospitals in southern Ethiopia
}

\author{
This article was published in the following Dove Press journal: \\ Drug Design, Development and Therapy \\ 10 August 2015 \\ Number of times this article has been viewed
}

\section{Temesgen Sidamo \\ Summoro' \\ Kassa Daka Gidebo² \\ Zewde Zemma Kanche' \\ Eskinder Wolka Woticha ${ }^{2}$ \\ 'School of Medicine, College of Health Sciences and Medicine, Wolaita Sodo University, Wolaita Sodo, Ethiopia; ${ }^{2}$ School of Public Health, College of Health Sciences and Medicine, Wolaita Sodo University, Wolaita Sodo, Ethiopia}

\begin{abstract}
Background: Rational prescribing is a primary step to ensure rational drug use. Often, half of the medicines are prescribed irrationally and half of these are even used incorrectly as the patients fail to take their medicines appropriately. The aim of this research was to evaluate drug-prescribing patterns of four hospitals in southern Ethiopia.

Methods: A retrospective cross-sectional study was conducted between May 15 and June 25, 2014, to evaluate the drug-prescribing patterns based on the World health Organization (WHO) prescribing indicators. The prescription papers, kept for the last 1 year in the outpatient departments of the four hospitals, were analyzed according to WHO guidelines. Also, prescriptions in the hospitals were analyzed to determine the most frequently prescribed drugs. All the statistical calculations were performed using SPSS ${ }^{\circledR}$ version 20.0 software.
\end{abstract}

Results and discussion: The average number of drugs per prescription ranges from $1.82 \pm 0.90$ to $2.28 \pm 0.90$, whereas the percentage of use of antibiotics and injections ranged from 46.7 to 85 and 15 to 61.7 , respectively. The average percentages of drugs prescribed by generic name and from the essential drugs list were 95.8 and 94.1, respectively. Anti-infective and analgesic drugs are found to be the most frequently prescribed medicines. In terms of polypharmacy, there was a slight deviation in prescribing patterns from what is acceptable according to the WHO criteria. Prescribing by generic name and from essential drug list was almost optimal. There was a significant deviation in the use of injectables in two of the four hospitals (50\%), whereas their use in the other two hospitals was within the acceptable range. The use of antibiotics in all the hospitals in present study was higher than the acceptable range.

Conclusion: Generally, it seems that there is need for improvement of the prescribing patterns in the hospitals, although this should be consolidated with further studies to link the patient diagnosis and the prescribed medications.

Keywords: prescribing indicators, medicines, hospitals, rational prescribing, Ethiopia

\section{Introduction}

The quality of prescribing is a major determinant of how patients use medicines. It plays a crucial role in the treatment of serious health conditions when people do tend to consult health professionals. The way in which health professionals prescribe multiple medicines reinforces consumers' beliefs that they need "a pill for every illness" and that a cure is unlikely without using medicines. It also affects the treatment of less severe conditions as people tend to remember the advice given and use it in later episodes of self-medication. ${ }^{1}$
Correspondence: Temesgen Sidamo Summoro

School of Medicine, College of Health Sciences and Medicine, Wolaita Sodo University, PO Box 138, Wolaita Sodo, Ethiopia

Tel +25I 911093567

Email teme.yene2003@gmail.com 
World health organization (WHO) has designed standardized prescribing indicators to evaluate the trends of prescribing in health facilities. These are the number of drugs per encounter, the percentage of drugs prescribed by brand name, the percentage of antibiotics and injectable drugs per encounter, and the percentage of drugs prescribed from an essential drug list (EDL). These indicators are used to describe current treatment practices, compare health facilities and prescribers, and allow for identification of potential drug-use problems that may affect patient care. ${ }^{1-3}$ The WHO recommended value of the number of medicines per prescription ranges from 1.4 to 1.8 , antibiotics prescription $20 \%$ to $27 \%$, and injectable medicines $13.4 \%$ to $24.1 \%$, whereas standard accepted value for prescribing by generic name and from EDL is $100 \%$. Based on these recommended values, drug-prescribing patterns in health institutions can be evaluated. Hence, based on an evaluation, the necessary modification to the pattern to achieve rational and cost-effective medication use may be suggested. A minimum of 600 patient records can be evaluated retrospectively for drug-prescribing pattern study in health facilities. ${ }^{1,3,4}$

All over the world, $50 \%$ of medications are prescribed inappropriately, and further, $50 \%$ of these are used inappropriately. ${ }^{4}$ Common irrational uses of drugs include over- or underuse of medications, high cost of the medications, indiscriminate and frequent use of injections and antibiotics, use of multiple medications, use of brand instead of generic names in prescribing, and prescribing medications not in accordance to a standard treatment guidelines or not from EDL are major problems of present-day medical practice. The consequences of these include ineffective treatment, development of antimicrobial resistance (AMR), adverse effects, and economic burden on patients and society. ${ }^{5,6}$

Whenever patients are exposed to multiple drugs, the incidence of adverse drug reactions increases. Thus, prescriptions with many drugs may be an indicator of inappropriate prescribing. It could be also an important index of the scope for educational intervention in rationalizing of prescribing practices. $^{6}$

Use of many antibiotics is another area where extravagant prescribing could be practiced. In developing countries, up to $75 \%$ of antibiotics are prescribed inappropriately. Such an irrational use of antibiotics fuels the AMR. AMR is growing for most major infectious diseases, including HIV/AIDS, tuberculosis, and malaria. ${ }^{4,6,7}$ The 2014 WHO report on global surveillance of AMR reveals that antibiotic resistance is no longer a prediction for the future; it is happening right now, across the world, and is putting at risk the ability to treat common infections in the community and hospitals. Appropriate use of antibiotics is necessary to prevent emergence of drugresistant bacteria as well as to minimize unnecessary wastage of scarce resource in developing countries. ${ }^{7}$

Also, overuse of injections is an irrational use of drugs. In developing countries, up to $56 \%$ of primary-care patients receive injections. From this, over $90 \%$ may be medically unnecessary. Globally, 15 billion injections are given, but half of these injections do not use sterilized needle and syringe, which may result in transmission of serious infectious diseases. ${ }^{8}$ Infections attributable to unsafe injection are hepatitis B (33\%), hepatitis C (42\%), HIV (2\%). Therefore, an urgent need arises to reduce injection use in developing countries to prevent health care-associated infections like HIV and other bloodborne pathogens. ${ }^{9}$

Rational drug use is achieved when there is rational prescribing using medicines from an EDL. Identifying a limited number of essential medicines leads to a better supply, more rational use, and lower costs. Drugs in the EDL are intended to fulfill the real needs of the majority of the population in diagnostic, prophylactic, therapeutic, and rehabilitative services using criteria such as risk-benefit ratio, cost-effectiveness, quality, practical administration, patient compliance, and acceptance. ${ }^{8,9}$

Studies conducted by members of the International Network for Rational Use of Drugs (INRUD) document health workers' practice of polypharmacy and people's use of drugs inappropriately. A study conducted in Indonesia found that the average number of drugs used to treat illnesses presented to the health worker was 3.8, both for children under 5 years and for the 5-years-and-above age group. Patients seemed to receive a similar mix of vitamins, analgesics, and antibiotics irrespective of their disorders. Also, in Philippines, it was observed that people keep prescriptions in their homes for reuse. ${ }^{10}$

Drug-prescribing patterns for outpatients were studied in three hospitals in northwest Ethiopia. According to the study, the average number of drugs per patient was reported to be 0.98 in Gondar, 1.8 in Bahir Dar, and 2.2 in Debre Tabor hospitals. Antibiotics (40\%-51\%) and analgesics (11\%-49\%) were also reported to be the most frequently prescribed drugs in these three hospitals. ${ }^{11}$ Few other drug-prescribing pattern studies were conducted at different times and in different hospitals and health facilities in Ethiopia. ${ }^{12-14}$ However, these are not comprehensive and consistent enough to identify the magnitude of problems related to irrational prescribing and drug use in order to take appropriate interventional measures to correct the trends. The primary aim of this study was to evaluate the prescribing patterns of medicines based on WHO indicators at the outpatient pharmacies of four hospitals: Wolaita Sodo University Teaching and Referral Hospital 
(WSUTRH), Tercha Zonal Hospital (TZH), Sodo Christian Hospital (SCH), and Dubo St. Mary's General Hospital (DSMGH), all of which are located in southern Ethiopia. The secondary aim was to evaluate prescriptions to identify the most frequently prescribed medications in the hospitals.

\section{Methods}

A cross-sectional study was carried out in the hospitals for the evaluation of drug-prescribing patterns starting from May 15 to June 25, 2014. Patient prescriptions kept for 1 year in the outpatient pharmacy of the hospitals between June 1, 2013 and May 25, 2014 were reviewed. Only those prescriptions with legible handwriting demonstrating all the essential components of a prescription were included. The prescription papers were selected by a systematic random sampling method to prevent data collection bias. In order to produce representative and comparable statistics of appropriate prescribing as per the definition of WHO, 360 prescription papers from each hospital (totally 1,440 prescriptions) within the specified period were utilized for this study. This accounts for $10 \%$ of the total prescriptions against the total annual prescriptions of 14,400 at the outpatient pharmacies of the hospitals. Also, 2,270 prescribed medications from the outpatient pharmacies of the two hospitals in Sodo town (WSUTRH and SCH) were analyzed with the aim of identifying the most frequently prescribed medications in the hospitals.

The data collection was carried out primarily by the outpatient pharmacists and pharmacy technicians in each hospital after the appropriate training and orientation. The data collection was supervised on a daily basis by the investigators involved in this study. Completeness of the data was checked every day during the data collection period. The data generated for each hospital were entered into a computer using
Statistical Package for the Social Sciences (SPSS) version 20.0 software (IBM Corporation, Armonk, NY, USA) to be edited, cleaned, and analyzed. The data were analyzed descriptively and summarized using tables and bar chart. The findings in this study were compared and contrasted with other national and international prescribing pattern studies. This research project was ethically cleared and approved by the institutional ethics review committee of the college of health sciences and medicine, Wolaita Sodo University.

\section{Results}

The main sociodemographic characteristics of patients obtained from prescriptions are shown in Table 1. The mean age of patients was $27.48 \pm 14.4$ years for the four hospitals as a whole. Male-to-female ratio was 0.75 , which indicates female patients' predominance. A total of 71 (4.9\%) patients were below 5 years of age. Majority of the patients were between the age of 15 and 44 years, which comprises $82.1 \%$ $(1,182)$, whereas patients between 45 and 64 years comprised $11.7 \%$ (169) of the total population. Elderly patients (age $>64$ ) account for $1.3 \%$ (18) of cases.

In total 2,999 drugs were prescribed for 1,440 patient encounters, making the overall average number of drugs per encounter $2.08 \pm 0.98$. The frequency of number of drugs per prescription and the average number of drugs per prescription in each hospital are summarized in Table 2. The other prescribing indicators, including the percentage of drugs prescribed by generic name, percentage of antibiotic drug encounter per prescription, percentage of encounter with injectable drugs per prescription, and percentage of drugs prescribed from EDL, are summarized separately in Figure 1. Prescriptions from two hospitals (WSUTRH and SCH) were also evaluated to identify the most frequently prescribed

Table I Sociodemographic characteristics of patients who visited $(N=I, 440)$ the four hospitals in southern Ethiopia, from June I I, 2013 to May 25,2014

\begin{tabular}{|c|c|c|c|c|c|}
\hline \multirow[t]{2}{*}{ Characteristics } & \multicolumn{4}{|l|}{ Hospitals } & \multirow[t]{2}{*}{ Total, N (\%) } \\
\hline & TZH, N (\%) & SCH, N (\%) & DSMGH, N (\%) & WSUTRH, N (\%) & \\
\hline \multicolumn{6}{|l|}{ Sex } \\
\hline Male & 173 (48.I) & $212(58.9)$ & II $3(31.4)$ & $120(33.3)$ & $618(42.9)$ \\
\hline Female & $187(5 \mid .9)$ & I 48 (4I.I) & $247(68.6)$ & $240(66.7)$ & $822(57.1)$ \\
\hline \multicolumn{6}{|l|}{ Age range (years) } \\
\hline$<5$ & $0(0.0)$ & $30(8.3)$ & $12(3.3)$ & $29(8.1)$ & 7I (4.9) \\
\hline $5-14$ & $84(23.3)$ & $32(8.9)$ & $36(10.0)$ & $25(6.9)$ & $177(12.3)$ \\
\hline $15-30$ & $180(50.0)$ & $126(35.0)$ & $243(67.5)$ & $168(46.7)$ & $717(49.8)$ \\
\hline $31-44$ & $72(20.0)$ & $80(22.2)$ & $60(16.7)$ & $76(2 I .1)$ & $288(20.0)$ \\
\hline $45-64$ & $24(6.7)$ & $84(23.3)$ & $9(2.5)$ & $52(14.4)$ & $169(11.7)$ \\
\hline$>64$ & $0(0.0)$ & $8(2.2)$ & $0(0.0)$ & $10(2.8)$ & $18(1.3)$ \\
\hline
\end{tabular}

Abbreviations: TZH, Tercha Zonal Hospital; SCH, Sodo Christian Hospital; DSMGH, Dubo St Mary's General Hospital; WSUTRH, Wolaita Sodo University Teaching and Referral Hospital. 
Table 2 Summary of the number of drugs per prescription, its frequency and percentage, and the average number of drugs per prescription in the four hospitals in southern Ethiopia $(\mathrm{N}=\mathrm{I}, 440)$, from June II, 2013 to May 25, 2014

\begin{tabular}{|c|c|c|c|c|}
\hline \multirow[t]{2}{*}{ Characteristics } & \multicolumn{4}{|l|}{ Hospitals } \\
\hline & SCH, N (\%) & DSMGH, N (\%) & TZH, N (\%) & WSUTRH, N (\%) \\
\hline \multicolumn{5}{|c|}{ Number of drugs per prescription } \\
\hline One & I44 (40.0) & $75(20.8)$ & $84(23.3)$ & $159(44.2)$ \\
\hline Two & $126(35.0)$ & $|4|(39.2)$ & I $44(40.0)$ & $132(36.7)$ \\
\hline Three & $62(17.2)$ & $114(31.7)$ & $84(23.3)$ & $47(13.1)$ \\
\hline Four & $18(5.0)$ & $30(8.3)$ & $48(13.3)$ & $20(5.6)$ \\
\hline Five & $4(1.1)$ & $0(0)$ & $0(0)$ & $2(0.6)$ \\
\hline Six & $6(1.7)$ & $0(0)$ & $0(0)$ & $0(0.0)$ \\
\hline \multicolumn{5}{|c|}{ Average number of drugs per prescription } \\
\hline & $1.97 \pm 1.06$ & $2.28 \pm 0.89$ & $2.27 \pm 0.97$ & $1.82 \pm 0.90$ \\
\hline
\end{tabular}

Note: Data is expressed as mean \pm standard deviation.

Abbreviations: TZH, Tercha Zonal Hospital; SCH, Sodo Christian Hospital; DSMGH, Dubo St Mary's General Hospital; WSUTRH, Wolaita Sodo University Teaching and Referral Hospital.

medications. A total of 2,270 prescribed medications from the two hospitals (WSUTRH $=1,437$ and $\mathrm{SCH}=833$ ) were analyzed to identify the most frequently prescribed medications is summarized in Table 3.

As it can be seen from Table 2, the averge number of drugs per patient was $1.97 \pm 1.06,2.28 \pm 0.89,2.27 \pm 0.97$, and $1.82 \pm 0.90$ for SCH, DSMGH, TZH, and WSUTRH, respectively. The percentages of prescriptions for more than two drugs were 25, 40, 36.6, and 19.3 for SCH, DSMGH, TZH, and WSUTRH, respectively. More than four drugs were not prescribed in two of the hospitals, DSMGH and $\mathrm{TZH}$, whereas $2.8 \%$ and $0.6 \%$ of the prescriptions contained five to six drugs in SCH, and four drugs in WSUTRH, respectively.

As indicated in Figure 1, the percentages of patients who received antibiotics were 46.7, 67.5, 85, and 66.7 in $\mathrm{SCH}$,
DSMGH, TZH, and WSUTRH, respectively. Also the percentages of injections were 57.2, 15, 16.7, and 61.7 in $\mathrm{SCH}$, DSMGH, TZH, and WSUTRH, respectively.

\section{Discussion}

\section{Level of polypharmacy}

The results indicated that except in case of extreme values, such as prescribing more than three drugs, cumulative average number of drugs per prescription $(2.08 \pm 0.98)$ is almost similar to the ideal value, which is less than $2 .{ }^{3}$ However, in two of the hospitals (50\%) more than two drugs were prescribed on average. The average number of drugs prescribed in this study is similar to a study conducted in private and public health centers in southwest Ethiopia. ${ }^{12}$ It is also nearly similar to what was reported by the Federal Ministry of Health (FMOH) of Ethiopia, ${ }^{13}$ Hawassa University

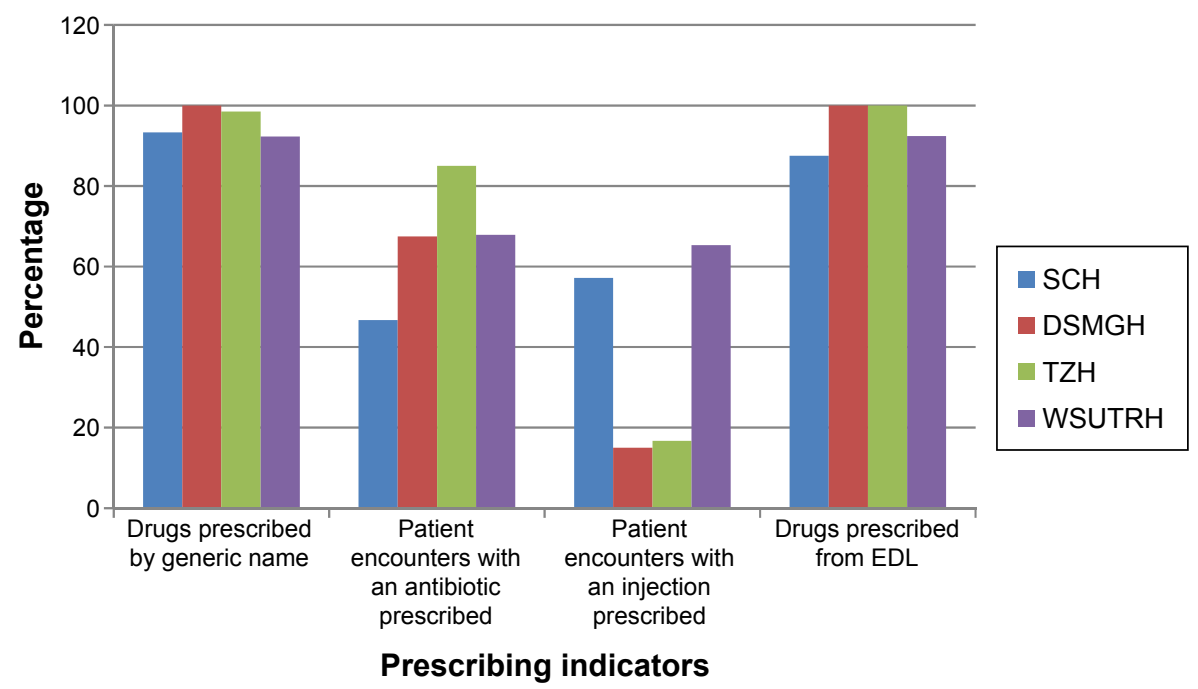

Figure I Comparison of some prescribing indicators in the four hospitals.

Abbreviations: SCH, Sodo Christian Hospital; DSMGH, Dubo St. Mary's General Hospital; TZH, Tercha Zonal Hospital; WSUTRH, Wolaita Sodo University Teaching and Referral Hospital; EDL, essential drugs list. 
Table 3 Distribution of the most frequently prescribed medications in two hospitals of southern Ethiopia from May 15 to June 25 , 2014

\begin{tabular}{|c|c|c|c|c|c|c|}
\hline \multirow[t]{3}{*}{ S/no } & \multicolumn{6}{|l|}{ Hospitals } \\
\hline & \multicolumn{3}{|l|}{ WSUTRH } & \multicolumn{3}{|l|}{ SCH } \\
\hline & Drugs prescribed & Frequency & Percentage & Drugs prescribed & Frequency & Percentage \\
\hline I & Amoxicilline & 184 & 12.8 & Tramadol & 96 & 11.5 \\
\hline 2 & Omeprazole & 140 & 9.7 & Diclofenac & 93 & 11.2 \\
\hline 3 & Tramadol & 129 & 9.0 & Ceftriaxone & 84 & 10.1 \\
\hline 4 & Ciprofloxacin & 128 & 8.9 & Metronidazole & 60 & 7.2 \\
\hline 5 & Metronidazole & 120 & 8.4 & Co-trimoxazole & 57 & 6.8 \\
\hline 6 & Ibuprofen & 92 & 6.4 & Paracetamol & 45 & 5.4 \\
\hline 7 & Cloxacillin & 79 & 5.5 & Ampicillin & 42 & 5.0 \\
\hline 8 & Diclofenac & 77 & 5.4 & Ibuprofen & 36 & 4.3 \\
\hline 9 & Doxycycline & 68 & 4.7 & Amoxicillin & 33 & 4.0 \\
\hline 10 & $\mathrm{CAF}$ & 64 & 4.5 & Normal saline & 30 & 3.6 \\
\hline II & Norfloxacin & 52 & 3.6 & Cloxacillin & 27 & 3.2 \\
\hline 12 & Prednisolone & 48 & 3.3 & Ciprofloxacin & 24 & 2.9 \\
\hline 13 & Cephalexin & 45 & 3.1 & Ferrous sulfate & 21 & 2.5 \\
\hline 14 & Furosemide & 36 & 2.5 & Multivitamin & 18 & 2.2 \\
\hline 15 & TTC & 25 & 1.7 & Cephalexin & 15 & 1.8 \\
\hline \multirow[t]{2}{*}{16} & Others & 150 & 10.4 & Others & 152 & 18.3 \\
\hline & Total & I,437 & 100 & Total & 833 & 100 \\
\hline
\end{tabular}

Abbreviations: WSUTRH, Wolaita Sodo University Teaching and Referral Hospital; SCH, Sodo Christian Hospital; CAF, chloramphenicol; TTC, tetracycline.

Teaching and Referral Hospital, ${ }^{14}$ and two teaching hospitals of Sudan, ${ }^{15}$ but much lower than that in India. ${ }^{16}$ Studies in Bangladesh ${ }^{17}$ and Lebanon ${ }^{18}$ reported that 1.4 and 1.6 drugs were prescribed per prescription, respectively. In this study, more than $30.2 \%$ of encounters (prescriptions) contained more than three drugs (minimum 3, maximum 6). This is much lower than a study in India, where $62.4 \%$ of prescriptions were with three or more drugs. ${ }^{19}$

The drug-prescribing patterns in WSUTRH and SCH show that antibiotics are the most frequently prescribed medications. This is consistent with the evaluation result of WHO prescribing indicator for antibiotic prescription encounters in this study. Approximately $60 \%$ of the top five drug prescriptions account for the antibiotics in the two hospitals (Table 3). In WSUTRH, three (amoxicillin, ciprofloxacin, and metronidazole) out of the top five (60\%) most frequently prescribed drugs were antibiotic medications. This is similar to a study in Hawassa University Teaching and Referral Hospital. ${ }^{14}$ Also, in $\mathrm{SCH}$, analgesic drugs (tramadol and diclofenac) constitute the top two frequently prescribed drugs (40\%), whereas the other three out of the top five drugs prescribed (60\%) were antibiotic medications. There is a general trend of prescribing more antibiotics and analgesic medications in the hospitals, as was evidenced in other similar study in northern Ethiopia. ${ }^{11}$ Tramadol is among one of the most frequently used analgesic medications in both SCH and WSUTRH, and for the question why this analgesic drug is prescribed most frequently, one of the doctors from WSUTRH stated that:

\footnotetext{
"Among the patients who visited our hospital there were many patients with an unknown sort of pain for which none of the laboratory diagnosis proved their causes. Such feelings of pain may be associated with a depressed psychological state of patients. Therefore, doctors usually prescribe analgesics like tramadol not only to alleviate a real pain. Rather they prescribe such medications for the psychology of the patients."
}

This may indicate that a significant number of patients visit the hospitals with nonspecific cases (for pain with unknown underlying causes), and the doctors usually prescribe analgesic pills for the satisfaction of the patients.

\section{Use of antibiotics}

Antibiotic encounters per prescription in this study range from $46.7 \%$ to $85 \%$ (the average was calculated to be $66.5 \%$ ). This is much higher than the ideal value recommended by WHO. ${ }^{3}$ It is even higher than what was reported by $\mathrm{FMOH}^{13}$ $(59 \%)$ and the three hospitals in northwest Ethiopia ${ }^{11}$ as well as those reported in most other countries such as Bangladesh, ${ }^{17}$ Lebanon, ${ }^{18}$ Nepal, ${ }^{20}$ and Tanzania, ${ }^{21}$ which ranges from $17.5 \%$ to $35.4 \%$. But the result is almost similar to that of the two teaching hospitals in Sudan which revealed $65 \%$ of antibiotic prescriptions per encounter. ${ }^{15}$ 


\section{Prescribing by generic name}

Writing prescriptions using generic name is an important drug-use quality to avoid undesirable drug interactions, adverse drug reactions, and medication errors. The average percentage of drugs prescribed by generic name in the four hospitals was 95.8. DSMGH and TZH exhibited the acceptable values in terms of the WHO criteria with regard to prescribing by generic names (almost $100 \%$ ), whereas it was found to be lower in SCH and WSUTRH. Private pharmacies might have influenced the prescribing patterns in later cases as they are relatively located in main city (Wolaita Sodo) where there are alternative private pharmacies, whereas the former are located in small towns. This can be explained by a reason given by a doctor from WSUTRH for a question, "why not all drugs are prescribed by generic name?" which was answered as "Some physicians who are working as part-time in private health facilities are more familiar with brand name than generic name".

But generally, in light of other local and international studies and according to the judgment criteria, the use of generic names in the hospitals in this study may be judged as a very good performance. For example, the study of southwest Ethiopia ${ }^{12}$ revealed that $92 \%$ of the drugs were prescribed by generic names, whereas those in Sudan ${ }^{15}$ and India ${ }^{16}$ indicated that only $43.6 \%$ and $73.4 \%$ of drugs were prescribed by generic name, respectively.

\section{Prescription of injectable dosage forms}

Injectable drug use in the four hospitals evaluated ranged between $15 \%$ and $61.7 \%$, whereas their average percentage was 37.7. Even though the values in two of the hospitals are within the acceptable range, the values in the other two hospitals and the average indicates that the proportion of patients who received injections is higher than the ideal value recommended by WHO, but the injection use is lower when compared with the study done in India, and higher than the study done in the Sudan, which was $10.5 \% .^{1,3,4,22}$

\section{Prescribing from national drug formulary}

Prescriptions in two of the four hospitals (50\%) exactly meet the standard criteria of prescribing medications from an EDL $(100 \%)$. However, the average value for the four hospitals $(94.1 \%)$ is short of meeting the WHO target level. It is also lower compared to another study report from southwest Ethiopia, which was $97 \% .{ }^{12}$ The relatively lower values in the other two hospitals (WSUTRH and SCH) account for the lowering of the overall average value. As these hospitals are located in the second biggest town in southern Ethiopia, other alternative medication use outlets (private pharmacies) and the drug consumption behavior of the people seem to influence the drug-prescribing patterns. Prescribing drugs from an EDL or national drug formulary is one of the indicators which measure rational drug use. , $22^{2}$

\section{Limitations}

Drug prescribing patterns in the present study were not linked with the diagnosis (indication) of the patients because such documents were not kept in the outpatient departments of the hospitals. If it were possible to link them, it would be easy to judge the degree of rationality of the drug prescriptions and their utilization. Association between the age of the patients and type of medications prescribed was also not made due to the same reason. If these were possible, it would be easy to judge the degree of rationality in prescribing patterns. However, we believe that this study can be used as a baseline for such comprehensive studies in the future, if patient diagnosis and medication history are documented appropriately.

\section{Conclusion and recommendations}

Generally, drug-prescribing practices of hospitals in this study need improvement in light of the WHO prescribing indicators. Particularly, the significant deviation from the acceptable WHO standard in prescribing antibiotics and injectable medications requires special attention. Use of many antibiotics may accelerate drug resistance, which is a global challenge that threatens the effective treatment of different infectious diseases. Also, overuse of injectable drugs may contribute to the spread of serious communicable diseases like HIV/AIDS and hepatitis. It seems that interventional measures are needed to improve the drug-prescribing patterns in these areas. However, since the indications and duration of treatments were not included in this study, further study is recommended to better judge the rationality/irrationality of use of much antibiotics and injectable drugs. Polypharmacy, use of brand names, and prescribing drugs that are not in the EDL for Ethiopia in present study were not exaggerated, though there was a slight deviation in comparison to the standard values.

\section{Acknowledgments}

We thank Wolaita Sodo University for funding this research. Also, we extend our heartfelt gratitude to the pharmacists, pharmacy technicians, and nurses working at Wolaita Sodo University Teaching and Referral Hospital, Tercha Zonal Hospital, Sodo Christian Hospital, and Dubo St. Mary's General Hospital for their kind assistance during data collection. 


\section{Disclosure}

The authors report no conflicts of interest in this work.

\section{References}

1. World Health Organization (WHO). Introduction to Drug Utilization Research. Oslo, Norway: WHO International Working Group for Drug Statistics Methodology, WHO Collaborating Centre for Drug Statistics Methodology, WHO Collaborating Centre for Drug Utilization Research and Clinical Pharmacological Services; 2003.

2. World Health Organization and University of Amsterdam. How to Investigate the Use of Medicines by Consumers. Geneva, Switzerland: World Health Organization and University of Amsterdam; 2004.

3. WHO. Action programme on essential drugs. How to Investigate Drug Use in Health Facilities. Geneva, Switzerland: WHO; 1993: 9-31.

4. WHO. Medicines use in primary care in developing and transitional countries. Facts Book Summarizing Results from Studies Reported between 1990 and 2006. Geneva, Switzerland: WHO; 2009.

5. Hardon A. Confronting Ill Health: Medicines, Self-Care and the Poor in Manila. Quezon-City, Philippines: Health Action Information Network; 1991.

6. Berger M, Murugi J, Buch E, et al. Strengthening pharmaceutical innovation in Africa. Council on Health Research for Development (COHRED); New Partnership for Africa's Development (NEPAD) 2009. Available from: http://policycures.org/downloads/ COHRED-NEPAD_Strengthening_Pharmaceutical_Innovation_ AfricaREPORT.pdf. Accessed November 27, 2014.

7. WHO. Antimicrobial Resistance, Global Report on Surveillance. Geneva, Switzerland: WHO; 2014. Available from: http://www.who.int/ drugresistance/documents/surveillancereport/en/. Accessed December $5,2014$.

8. Jonathan DQ. Essential Drugs and Medicines Policy. Geneva, Switzerland: World Health Organization International Paediatrics Association; 2001.

9. Management Sciences for Health. Managing Drug Supply. 2nd ed. West Hartford, CT: Kumarian Press; 1997.

10. The Academy of Managed Care Pharmacy's. Drug Use Evaluation, Alexandria, VA: The Academy of Managed Care Pharmacy's; 2008. Available from: http://www.amcp.org/workarea/downloadasset. aspx?id=9296. Accessed August 12, 2011.
11. Desta Z, Abula T, G.yohannes A, Worku A. Drug prescribing patterns for outpatients in three hospitals in North West Ethiopia. Ethiop J Health Dev. 2002;16(2):183-189.

12. Tsega B, Hailu W, Ergetie Z. Measuring quality of drug use in primary health care facilities: a yearlong assessment of WHO prescribing indicators, Wolkite town, South West Ethiopia. Int J Pharm Ind Res. 2012; 2(04):485-491.

13. Federal Democratic Republic of Ethiopia, Ministry of Health. Assessment of the Pharmaceutical Sector in Ethiopia. Addis Ababa, Ethiopia: Federal Democratic Republic of Ethiopia; 2003.

14. Desalegn A. Assessment of drug use pattern using WHO prescribing indicators at Hawassa University teaching and referral hospital, south Ethiopia: a cross-sectional study. BMC Health Serv Res. 2013; 13:170.

15. Awad AI, Himad HA. Drug use practice in teaching hospitals of Khartoum State, Sudan. Eur J Clin Pharmacol. 2006;62(12): 1087-1093.

16. Karande S, Sankhe P, Kulkarni M. Patterns of prescription and drug dispensing. Indian J Pediatr. 2005;72:117-121.

17. Guyon AB, Barman A, Ahmed JU, Ahmed AU, Alam MS. A baseline survey on use of drugs at the primary health care level in Bangladesh. Bull WHO. 1994;72:265-271.

18. Hamadeh GN, Dickerson LM, Saab BR, Major SC. Common prescriptions in ambulatory care in Lebanon. Ann Pharmacother. 2001; 35:636-640.

19. Ravi Shankar P, Partha P, Nagesh S. Prescribing patterns in medical outpatients. Int J Clin Pract. 2002;56:549-551.

20. Ghimire S, Nepal S, Bhandari S, Nepal P, Palaian S. A prospective surveillance of drug prescribing and dispensing in a teaching hospital in Western Nepal. J Pak Med Assoc. 2009;59:726-731.

21. Massele AY, Nsimba SE, Rimoy G. Prescribing habits in church-owned primary health care facilities in Dar Es Salaam and other Tanzanian coast regions. East Afr Med J. 2001;78:510-514.

22. Hutin YJF, Hauri AM, Armstrong GL. Use of injections in healthcare setting worldwide, 2000: literature review and regional estimates. $B M J$. 2003;327:1075-1079.

\section{Publish your work in this journal}

Drug Design, Development and Therapy is an international, peerreviewed open-access journal that spans the spectrum of drug design and development through to clinical applications. Clinical outcomes, patient safety, and programs for the development and effective, safe, and sustained use of medicines are a feature of the journal, which

\section{Dovepress}

has also been accepted for indexing on PubMed Central. The manuscript management system is completely online and includes a very quick and fair peer-review system, which is all easy to use. Visit http://www.dovepress.com/testimonials.php to read real quotes from published authors. 